\title{
Cumulative Adversity Sensitizes Neural Response to Acute Stress: Association with Health Symptoms
}

\author{
Dongju Seo', Kristen A Tsou', Emily B Ansell', Marc N Potenza ${ }^{1,2,3}$ and Rajita Sinha*, ${ }^{*, 2,3}$ \\ 'Department of Psychiatry, Yale University School of Medicine, New Haven, CT, USA; ${ }^{2}$ Department of Neurobiology, Yale University School of \\ Medicine, New Haven, CT, USA; ${ }^{3}$ Department of Child Study, Yale University School of Medicine, New Haven, CT, USA
}

\begin{abstract}
Cumulative adversity (CA) increases stress sensitivity and risk of adverse health outcomes. However, neural mechanisms underlying these associations in humans remain unclear. To understand neural responses underlying the link between CA and adverse health symptoms, the current study assessed brain activity during stress and neutral-relaxing states in 75 demographically matched, healthy individuals with high, mid, and low CA (25 in each group), and their health symptoms using the Cornell Medical Index. CA was significantly associated with greater adverse health symptoms $(P=0.01)$ in all participants. Functional magnetic resonance imaging results indicated significant associations between CA scores and increased stress-induced activity in the lateral prefrontal cortex, insula, striatum, right amygdala, hippocampus, and temporal regions in all 75 participants $(p<0.05$, whole-brain corrected). In addition to these regions, the high vs low CA group comparison revealed decreased stress-induced activity in the medial orbitofrontal cortex (OFC) in the high CA group $(p<0.01$, whole-brain corrected). Specifically, hypoactive medial OFC and hyperactive right hippocampus responses to stress were each significantly associated with greater adverse health symptoms $(p<0.0 \mathrm{I})$. Furthermore, an inverse correlation was found between activity in the medial OFC and right hippocampus $(p=0.0 \mathrm{I})$. These results indicate that high CA sensitizes limbic-striatal responses to acute stress and also identifies an important role for stress-related medial OFC and hippocampus responses in the effects of CA on increasing vulnerability to adverse health consequences.

Neuropsychopharmacology (2014) 39, 670-680; doi:10.1038/npp.2013.250; published online 16 October 2013
\end{abstract}

Keywords: cumulative adversity; health problems; hippocampus; orbitofrontal cortex

\section{INTRODUCTION}

Cumulative adversity (CA) refers to repeated, adverse social and environmental events that an individual experiences throughout a lifetime (Thoits, 2010; Turner et al, 1995). Accumulation of these adverse exposures throughout one's lifetime are conceptualized as independent of subjective perception of stress and, thus, may represent an 'allostatic' load, which may increase subsequent vulnerability to mental and physical disorders (Thoits, 2010). Stress and adversity increases risk for morbidity and mortality (Seeman et al, 2004), and a strong link between psychosocial stress and biological dysregulation in various physical domains is well documented (McEwen and Stellar, 1993; Seeman et al, 2004). For example, individuals with a history of trauma and environmental adversity are at a greater risk of psychiatric and other chronic diseases (Lloyd and Turner, 2008; Thoits, 2010). Thus, a significant literature has accrued identifying the biological factors influenced by high $\mathrm{CA}$, including progressive alterations in neuro-

*Correspondence: Professor R Sinha, Department of Psychiatry, Neurobiology and Child Study, Yale University School of Medicine, 2 Church Street South, Suite 209, New Haven, CT 06519, USA,

Tel: + | 203737 5805, Fax: + | 203737 1272,

E-mail: Rajita.sinha@yale.edu

Received 16 January 2013; revised 13 September 2013; accepted 14 September 2013; accepted article preview online 20 September 2013 endocrine, autonomic, cardiovascular, and inflammatory responses (McEwen, 1998; McEwen and Stellar, 1993; Seeman et al, 2004). However, brain mechanisms underlying the link between high CA and its associated health risk in humans remain unclear.

Multiple studies have shown adverse effects of repeated and high chronic stress on the prefrontal cortex (PFC) and limbic-striatal function. Animal studies have shown repeated stress-related compromised function in the prefrontallimbic-striatal regions, including dendritic damage in the medial PFC (Radley et al, 2006b), reduced GABA-stimulated chloride uptake in the amygdala (Martijena et al, 2002), altered synaptic structure in the hippocampus (Karst and Joels, 2003), and upregulated striatal function (Rossi et al, 2008). Specifically, the PFC, a crucial region for stress and emotion regulation ( $\mathrm{Li}$ and Sinha, 2008), is found to be hypoactive during post stress cognitive manipulation (Liston et al, 2006; Ossewaarde et al, 2011). Disrupted prefrontal attentional control during acute stress was also found in healthy individuals (Liston et al, 2009).

In relation to physical health, the prefrontal-limbicstriatal region is involved in the modulation of physiological pain (Borsook et al, 2007), autonomic balance (Thayer et al, 2012), endocrine arousal (Diorio et al, 1993; Figueiredo et al, 2003), and immune responses (Phillips et al, 1999), suggesting that dysregulation of this circuit could have adverse health effects in vulnerable individuals even 
without physical or psychological disorders. These studies emphasize the need to investigate the functional role of this circuit in response to acute stress in the association between high CA and health problems in humans. To understand the neural responses that modulate $\mathrm{CA}$ and adverse health symptoms, the current study utilized functional magnetic resonance imaging (fMRI) to assess brain responses to acute stress $v s$ non-stressful, neutral-relaxing scenarios in 75 healthy individuals representing a full range of CA scores. For the CA assessment, we used the Cumulative Adversity Interview (CAI; Turner et al, 1995), a wellvalidated measure shown to be predictive of physical and mental health problems in large population-based studies (Ansell et al, 2012a,b; Lloyd and Turner, 2008; Turner and Gil, 2002; Turner and Lloyd, 2004). To specifically identify neural substrates pertaining to CA-related vulnerability for adverse health consequences, we utilized extreme group comparisons by contrasting the high vs low CA groups, based on the previous evidence that such designs increase statistical power and show sensitivity to detect risk patterns with greater predictive validity (Abrahams and Alf, 1978; Angold et al, 2002; Fowler, 1992; Henshall and Goddard, 1999; Romens et al, 2009). In addition, healthy individuals with the intermediate range of CA were included to ensure a full range of continuous CA scores in the sample. The high, mid and low CA groups were demographically matched and were a representative subset of a large community cohort assessed on CA. Current adverse physical and psychological health symptoms were measured using the Cornell Medical Index (CMI; Abramson, 1966). We also utilized the wellestablished, individualized script-driven imagery method for a brief induction of stress and neutral-relaxing experiences (Sinha, 2009) to account for individual variation in stress responses (Stroud et al, 2002). On the basis of previous research cited above, we expected that CA would be associated with brain response to acute stress in the prefrontal-limbic-striatal circuitry. We also hypothesized that individuals with high CA would show decreased prefrontal regulatory function and increased stress-induced reactivity in limbic-striatal regions, and this pattern of response would be associated with current health symptoms as measured by the CMI.

\section{MATERIALS AND METHODS}

\section{Participants}

Participants were derived from a sample of 419 community individuals recruited via local newspaper and advertisements for research participation (see Supplementary Methods, for details). All subjects completed the CAI structured interview and were further screened for handedness and interest in participating in a neuroimaging study. A sample of 75 , right-handed healthy individuals with high (top 30\%), medium (middle 30-70\%), or low (bottom 30\%) total scores on the CA (25 in each group, demographically matched) participated in an fMRI session. Participants also completed psychiatric, cognitive, demographic, and health assessments, including the CMI and medical evaluations to ensure good health status (see Supplementary Information). All research staff interacting with study participants (including the administration of CAI and CMI) were blinded to study aims and hypotheses. All participants were free of any mental disorders verified by the Structured Clinical Interview for DSM-IV, and no participants were on any medications at the time of fMRI testing. All study procedures were approved by the Human Investigation Committee at the Yale University School of Medicine, and all participants signed an informed consent prior to study participation.

\section{Cumulative Adversity Interview}

The CAI is a semistructured interview that assesses 140 events encompassing childhood and adult trauma, recent or major adverse life events, and chronic stress including events pertaining to violence, death and loss of loved ones, natural disasters, work/job, school and education, finance/ income, relationship and marital status, and living environment (Turner et al, 1995). The CAI consists of four subscales: three subscales related to the objective count of CA (major life events, recent life events, and life trauma) resulting from both external forces and subjects' own behaviors and a chronic stress subscale pertaining to subjective response to stress and adversity (for details, see Supplementary Methods). In order to detect neural activity only related to objective CA events, the current study excluded the chronic stress measure and focused only on the CA scores, which are derived from the sum count of number of adverse life events from recent life events, major life events, and trauma that have occurred over the lifespan (see Supplementary Table S1). Recent evidence from our laboratory indicated high CA scores were associated with lower gray matter volume in the medial PFC, insula, and striatal regions (Ansell et al, 2012b). The CAI has also been shown to be predictive of mental health disorders and physical health conditions in large population-based studies (Brown and Turner, 2010; Gayman et al, 2008; Russell et al, 2009; Scott et al, 2008; Turner and Lloyd, 2004).

\section{Cornell Medical Index}

The CMI captures current physical and psychological health symptoms presented in 195 questions in 18 sections (Abramson, 1966; see Supplementary Methods and Supplementary Table S2) and has been validated as a good indication of general health in many studies (Costa and McCrae, 1985; Perlmutter and Nyquist, 1990).

\section{Individualized Imagery Method and Efficacy of Imagery Manipulation}

The script-driven individualized imagery method is a wellestablished validated method for brief provocation of emotions, acute stress, and anxiety states in laboratory and neuroimaging studies (Britton et al, 2005; Jastreboff et al, 2013; Orr et al, 1993; Potenza et al, 2012; Seo et al, 2013; Sinha et al, 2004; see Supplementary Methods for further description).

Before the fMRI session, individually customized imagery scripts were developed based on participants' reports of two stressful and two neutral-relaxing experiences using the standardized Scene Construction Questionnaires (for detailed method see Sinha, 2009). For stress scripts, participants described a situation that made them sad, mad, and 
upset that could not be changed in the moment (eg, being fired, family discord, or relationship conflict). The severity of the situations were rated by the participants on a 10 point Likert scale $(1=$ not at all stressful and $10=$ the most stressful), using only events rated as 8 or above for stimulus provocation and script development. Neutral-relaxing scripts pertained to personal experiences of neutral-relaxing situations (eg, reading in a park or watching the waves at the beach). Each script was standardized across conditions and subjects in terms of script style, content format, and length, while preserving the individual stimulus and response descriptors, as described previously (Sinha, 2009), and then audiotaped for script presentation. Each 2-min audiotaped script was presented in random order during the scanning session.

In order to ensure efficacy of the imagery manipulation, imagery ability of participants were assessed using the Questionnaire on Mental Imagery (QMI; Sheehan, 1967). There was no statistical difference in QMI scores and in post-imagery ratings of vividness among the high, mid, and low CA groups, suggesting equivalent levels of imagery ability and task performance (see Supplementary Methods for detailed results and description). Before the scanning session, a standardized relaxation and imagery training procedure (Sinha, 2009) was implemented in all participants to minimize variability in imagery ability.

\section{fMRI Acquisition, Task, and Physiological and Behavioral Anxiety Measures}

A 3-T Siemens Trio MRI system with a single-channel standard quadrature head coil was utilized to acquire MRI imaging data using a $\mathrm{T} 2{ }^{\star}$-sensitive gradient-recalled singleshot echo-planar pulse sequence (see Supplementary Information for fMRI parameters). Four fMRI trials were acquired, each lasting $5 \mathrm{~min}$. It consists of a 1.5 -min silent baseline followed by a 2.5 -min imagery $(2 \mathrm{~min}$ of readimagery and $0.5 \mathrm{~min}$ of quiet-imagery) and a 1 -min silent recovery period. Baseline period consisted of lying still with no mental activity, and the recovery period entailed stop imagining and staying still in the scanner. Across subjects, order of script condition was counterbalanced to control for order effects and then the condition order was randomly assigned to each subject. Each script was presented only once without the same condition presented consecutively. Before and after each fMRI trial, behavioral ratings for anxiety were collected using a 10 -point verbal scale $(1=$ not at all and $10=$ extremely high). Participants were instructed to rate how tense, anxious, and/or jittery they felt at that moment. Continuous measures of pulse during each trial were obtained using a pulse oximeter placed on the subject's non-dominant forefinger. All subjects participated in a 2min progressive relaxation between fMRI trials to stabilize any residual anxiety and arousal from prior trials.

\section{fMRI Analysis}

fMRI data were converted from Digital Imaging and Communication in Medicine format to Analyze format using XMedCon (Nolfe, 2003). To reach a steady-state equilibrium between radio-frequency pulsing and relaxation, the first 10 images were removed from each functional run. The recovery period ( $1 \mathrm{~min}$ ) was excluded due to potential carryover effects from the imagery period. Using MATLAB and Statistical Parametric Mapping (SPM5), fMRI data were preprocessed with slice time correction and motion correction for three translational and three rotational directions, discarding any trial with linear motion $>1.5 \mathrm{~mm}$ and a rotation exceeding $2^{\circ}$. General linear model (GLM) was used for individual level analysis on each voxel in the entire brain volume with a regressor that compares time during imagery to the baseline for each trial per condition (stress-baseline and neutral-baseline) using BioImageSuite (Duncan et al, 2004). In order to account for any variability in baseline fMRI signal, drift correction was implemented in the GLM and drift regressors were used to remove the mean time course, linear, quadratic, and cubic trends for each functional run. Each trial was then spatially smoothed using a $6-\mathrm{mm}$ Gaussian kernel and individually normalized to generate $\beta$ maps $(3.44 \mathrm{~mm} \times 3.44 \mathrm{~mm} \times 4 \mathrm{~mm})$. To account for individual anatomical differences, three sequential registrations were applied to the individual normalized $\beta$-maps using BioImageSuite (Duncan et al, 2004): (1) linear registration between the individual subjects' functional image to the T1 structural image (within subject), (2) linear registration between the T1 structural image and the 3D MPRAGE image $(1 \times 1 \times 1 \mathrm{~mm})$, and (3) non-linear registration to a reference 3D image. The reference image was the Colin27 Brain (Holmes et al, 1998), a high-definition anatomical image registered to the Montreal Neurological Institute space.

The second-level group analysis was conducted with BioImageSuite and Analysis of Functional NeuroImages software (AFNI) utilizing random mixed effects models. In order to examine the association between $\mathrm{CA}$ and brain activity in all 75 individuals, a whole-brain correlational analysis was implemented using BioImageSuite. For the high $v s$ low CA group comparison, a $2 \times 2$ ANOVA (group by condition) was carried out with condition (neutral/ stress) as the within-subjects fixed-effect factor, group (high/low CA) as the between-subjects factor, and subject as the random-effect factor. To correct for multiple comparisons, we used cluster-wise control of family-wise errors; the $t$-value and correlation maps were cluster corrected at $p<0.05$ and $p<0.01 \quad(p<0.05$ voxel-wise threshold and $3537 \mathrm{~mm}^{3}$ at a cluster-level significance $\alpha<0.05$ through Monte Carlo simulation; $p<0.01$ voxel-wise threshold and $1134 \mathrm{~mm}^{3}$ at a cluster-level significance $\alpha<0.01$; two-tailed). The minimum cluster size was determined by Monte Carlo simulation (Xiong et al, 1995) using the AFNI AlphaSim program on voxels within the gray matter $\left(586710 \mathrm{~mm}^{3}\right)$. To best identify relevant neural substrates and be appropriately conservative, a threshold of 0.05 was used for whole-brain correlation analyses and 0.01 was applied for group difference maps.

\section{RESULTS}

\section{Sample Description}

Table 1 summarizes the demographic characteristics and CAI scores for the sample. There were no significant differences in age, education, gender, race, employment, economic status, and scores of State-Trait Anxiety Inventory (Spielberger et al, 1983) among the three CA groups, 
Table I Demographics and Health Characteristics.

\begin{tabular}{|c|c|c|c|}
\hline Subject variable & $\begin{array}{c}\text { High CA } \\
N=25\end{array}$ & $\begin{array}{c}\text { Mid CA } \\
N=25\end{array}$ & $\begin{array}{c}\text { Low CA } \\
N=25\end{array}$ \\
\hline \multicolumn{4}{|l|}{ Demographics } \\
\hline Age (years) & $28.84(8.9)$ & $26.56(7.9)$ & $27.44(7.6)$ \\
\hline Gender, female (\%) & $9(36.0)$ & $5(20.0)$ & $9(36.0)$ \\
\hline Smoker (\%) & $4(16.0)$ & $3(12.0)$ & $4(16.0)$ \\
\hline Race, Caucasian (\%) & $17(68.0)$ & $17(68.0)$ & $17(68.0)$ \\
\hline Education & $15.24(1.9)$ & $15.24(1.9)$ & $15.16(2.5)$ \\
\hline Body mass index & $28.31(5.9)$ & $26.33(4.7)$ & $27.52(4.5)$ \\
\hline $\begin{array}{l}\text { Employment status }{ }^{\mathrm{a}} \text {, } \\
\text { employed (\%) }\end{array}$ & $22(88)$ & $25(100)$ & $23(92)$ \\
\hline $\begin{array}{l}\text { Average income, past } \\
30 \text { days }\end{array}$ & $1211.6(1774.6)$ & 925.1 ( 1655.2$)$ & I $125(\mid 279.1)$ \\
\hline \multicolumn{4}{|l|}{ STAI scores } \\
\hline Total score & $22.64(5.6)$ & $22.80(5.5)$ & $20.96(4.1)$ \\
\hline STAl state anxiety & $31.08(8.2)$ & $32.32(8.5)$ & $30.68(7.5)$ \\
\hline STAI trait anxiety & $32.0(8.6)$ & $32.28(8.0)$ & $32.52(10.4)$ \\
\hline \multicolumn{4}{|l|}{ CAl scores ${ }^{\mathrm{b}}$} \\
\hline CA life event**** & | $4.84(3.0)$ & $7.64(1.5)$ & $3.36(1.5)$ \\
\hline Major life events***** & $2.64(1.4)$ & $1.64(1.3)$ & $0.44(0.7)$ \\
\hline Recent life events***** & $3.88(1.9)$ & $2.4(1.8)$ & $0.48(0.7)$ \\
\hline Trauma****** & $8.32(3.4)$ & $3.6(2.0)$ & $2.44(1.3)$ \\
\hline \multicolumn{4}{|l|}{ CMI scores ${ }^{c}$} \\
\hline Total* & | $3.44(\mid 1.6)$ & $10.8(8.3)$ & $6.2(4.8)$ \\
\hline Physical health*** & $10.04(8.2)$ & $7.5(4.9)$ & $4.6(3.3)$ \\
\hline Psychological health & $3.4(4.6)$ & $3.3(5.0)$ & $1.6(2.3)$ \\
\hline
\end{tabular}

Abbreviations: BMI, body mass index; CA, cumulative adversity; CAI, Cumulative Adversity Interview; CMI, Cornell Medical Index; STAI, State-Trait Anxiety Inventory.

Mean values (SD) are denoted for age, education years, BMI, income, STAl, CAl, and $\mathrm{CMI}$ scores. All other measures reported in frequency (percents). There is no group difference in demographics and STAl anxiety scores. Asterisks show significant group differences. ${ }^{*} * * * 0001$; ${ }^{*} * p<0.0$ I; $* p<0.05$.

${ }^{a}$ Individuals with full-time/regular part-time employment and students.

${ }^{b}$ See Supplementary Table SI for the list of the CAI adverse life events.

'See Supplementary Table S2 for CMI health symptoms and the frequency with which each was endorsed.

except for CAI and CMI scores. The score of CA life event was positively correlated with CMI health problems $(r=0.29, p=0.01)$, with no outliers in all 75 individuals.

\section{Experimental Manipulations: Anxiety and Heart Rate}

Behavioral data analysis was conducted to examine the effects of experimental manipulation and their associations with CA. Given the continuous nature of CA levels in 75 individuals, heart rate and anxiety ratings were examined using Student's $t$-tests between stress and neutral conditions in all subjects for an experimental manipulation check (Figure 1). Next, correlation analyses were conducted to examine the associations between CA and these measures. During stress exposure, both anxiety $(t=9.37, p<0.0001)$ and heart rate $(t=5.13, p<0.0001)$ were significantly elevated during stress exposure compared with the neutral condition, indicating successful stress induction. Heart rate response was positively correlated with CA scores during both the neutral $(r=0.24, p<0.05)$ and stress $(r=0.25$, $p<0.05)$ conditions in all 75 individuals with no outliers. Self-reported anxiety ratings were not correlated with CA.

\section{fMRI Results}

Correlation with CA. Whole-brain correlation analyses indicate significant positive correlations between stressinduced brain activity (stress-baseline) and CA scores in the prefrontal-limbic-striatal circuit in all 75 participants, including bilateral $\mathrm{PFC}$, insula, striatum, right amygdala, hippocampus, temporal gyrus, right thalamus, and cerebellum (Figure 2 and Supplementary Table S3; whole-brain corrected at $p<0.05$ ). During neutral trials (neutral-baseline), one cluster involving the left parietal lobe (precuneus and inferior parietal lobe (IPL)), was found to be associated with CA (Supplementary Table S3). There were no outliers in any of these associations. The scatter plot in Figure 2 illustrates positively correlated patterns in areas of the lateral PFC, right amygdala, and striatum during stress.

High and low CA groups on stress/neutral responses. Significant group difference was also found between high and low CA individuals in brain response to stress in prefrontal-limbic-striatal regions (Figure 3 and Supplementary Table S4; whole-brain FWE corrected at $p<0.01$ ). During stress exposure (stress-baseline), high CA individuals showed increased activity in the lateral PFC, insula, right amygdala and hippocampus, striatum (putamen and ventral striatum), midbrain, posterior cingulate cortex, and temporal and parietal lobe, but decreased activity in the medial orbitofrontal cortex (OFC) compared with low CA individuals. No group differences during the neutral condition survived correction for multiple comparisons.

Neural link between CA and CMI health symptoms. To identify CA-related neural responses that may be associated with CMI health symptoms, mean signal changes in significant brain activation regions involved in CA (correlation and group difference maps) were independently correlated with the CMI scores. Among these regions of interests, only the medial OFC and right hippocampus from the high vs low CA group difference map (Figure 3) was significantly associated with CMI health problems (Figure 4; also see Supplementary Figure $\mathrm{S} 1$ for $\mathrm{OFC}$ and right hippocampus response in high, mid, and low CA groups). In $50 \mathrm{high} /$ low CA individuals, medial OFC activity was negatively correlated with CMI total scores $(r=-0.42$, $p<0.01$ ), as well as with subscale scores of physical $(r=-0.38, \quad p<0.01)$ and psychological $(r=-0.39$, $p<0.01)$ health symptoms. Right hippocampal activity was positively correlated with CMI total scores $(r=0.37$, $p<0.01)$, as well as scores of physical $(r=0.31, p<0.05)$ and psychological health $(r=0.39, p<0.01)$ symptoms. Furthermore, medial OFC activity was inversely correlated with the right hippocampal activity $(r=-0.36, p=0.01)$. No outliers were found in any of these associations. When 
a

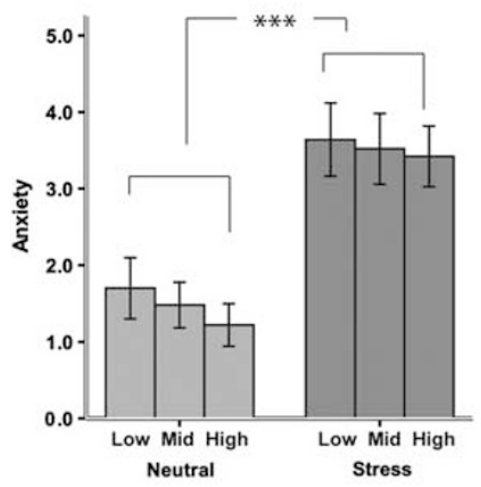

b

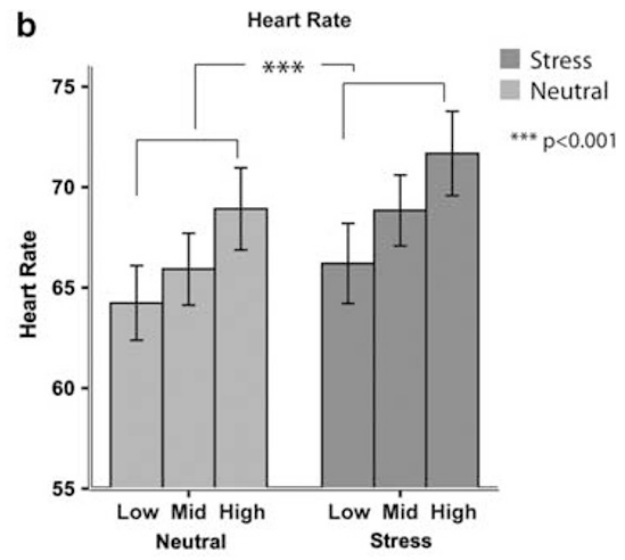

C

Heart Rate \& Cumulative Adversity
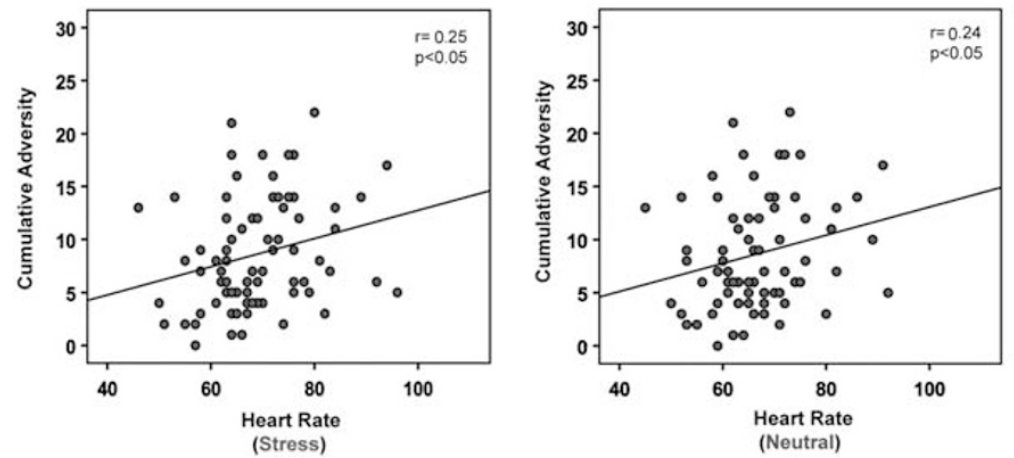

Figure I (a) Anxiety ratings; (b) heart rate responses during stress and neutral conditions; and (c) scatterplots showing significant correlations between cumulative adversity (CA) and heart rate during stress and neutral conditions in all 75 participants. During stress exposure, (a) anxiety ratings ( $t=9.37$, $p<0.000 \mathrm{I})$ and $(\mathrm{b})$ heart rate $(t=5.13, p<0.000 \mathrm{I})$ were significantly elevated relative to the neutral condition in all participants. (c) Heart rate response was correlated with CA score in both neutral $(r=0.24, p<0.05)$ and stress $(r=0.25, p<0.05)$ conditions. No correlation was found between anxiety ratings and CA. Error bar indicates SEM. ****P $<0.0001$.

these two ROIs were applied to all 75 subjects, the associations with CMI health problems remained significant in both medial OFC $(r=-0.28, p=0.016)$ and right hippocampus $(r=0.25, p<0.05)$ activity with a trend-level inverse relationship between the medial OFC and right hippocampus $(r=-0.21, p<0.07)$ in all 75 subjects.

\section{DISCUSSION}

We investigated neural responses to acute stress and its association with CA and health symptoms in a community sample of individuals, without psychiatric or physical diseases. In all participants, CA was significantly associated with increased activity in regions of the lateral PFC, insula, striatum, right amygdala, and hippocampus during stress exposure, and in the left parietal lobe during the neutral condition. When the high CA was directly compared with the low CA group, significant differences were also found in these key prefrontal-limbic-striatal regions during stress exposure. In addition, decreased activity in the medial OFC was specifically revealed in the high vs low CA group comparison. Among these CA-related brain activation regions, medial OFC and right hippocampal activity during stress exposure were inversely correlated and found to be associated with CMI-assessed health problems.

In subcortical response to acute emotional stress, significant associations with CA were mainly found in limbic-striatal regions, including the putamen, amygdala, hippocampus, and the insula cortex, indicative of a sensitized neural response to acute stress in individuals with high CA. Limbic-striatal activation has been associated with emotions and stressful experiences. During the experience of stress or aversive emotion, the amygdala is activated to initiate the stress response, including the release of corticotropin-releasing hormone and norepinephrine (Panksepp et al, 1997; Sinha, 2008), and interacts with the hippocampus for accessing emotional memory (Phelps, 2004). Limbic activity was predominantly found in the right hemisphere, consistent with the role of rightlateralized limbic activity in the processing of negative emotions (Lanteaume et al, 2007; Morris et al, 1999), suggesting sensitized right limbic responses during emotional stress in individuals with high CA. Along with amygdala-hippocampal regions, increased activity in the insula and striatum was evident. The insula is involved in emotional arousal and interoceptive awareness (Craig, 2009). The striatum has been associated with habitual responses to emotional stimuli (Schultz, 2006) and modulation of impulsive behaviors (Vink et al, 2005). Activation in the insula and striatum during acute stress has been reported in healthy individuals (Seo et al, 2011). In rats, chronic stress exposure upregulates and compromises striatal function (Rossi et al, 2008), and influences prefrontal executive function by altering dorsal striatal 

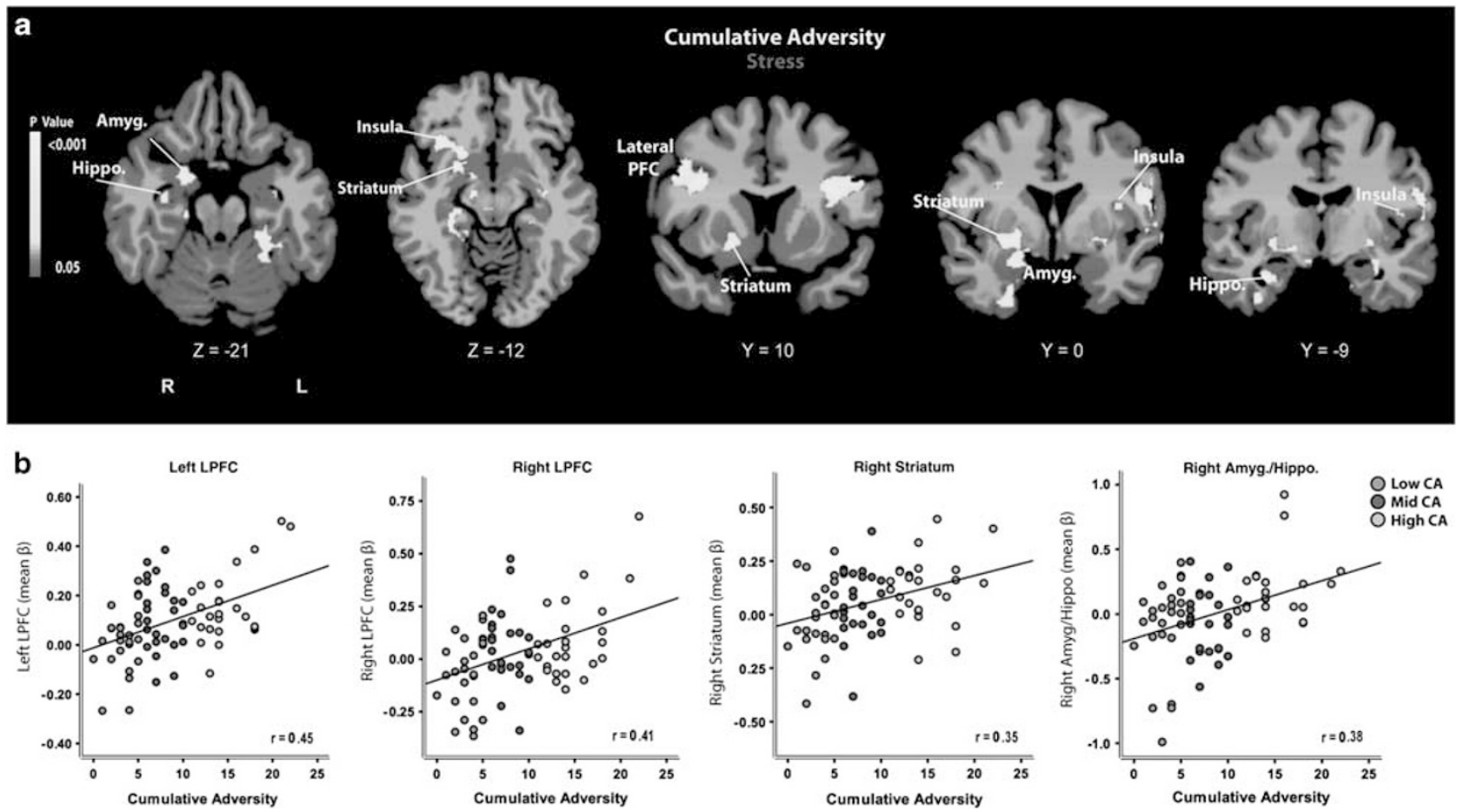

O Low CA

O High CA

Figure 2 Results of whole-brain voxel-based correlation analysis showing associations between stress-induced brain activity and cumulative adversity (CA) scores. In all 75 healthy individuals, (a) CA was positively correlated with activity in the bilateral prefrontal cortex (PFC), insula, striatum, right amygdala, and hippocampus during stress exposure (whole-brain FWE-corrected, $p<0.05$ ). (b) Scatterplots further illustrate the correlated pattern in these regions with no outliers. L, left; R, right. Montreal Neurological Institute (MNI) coordinates were used.
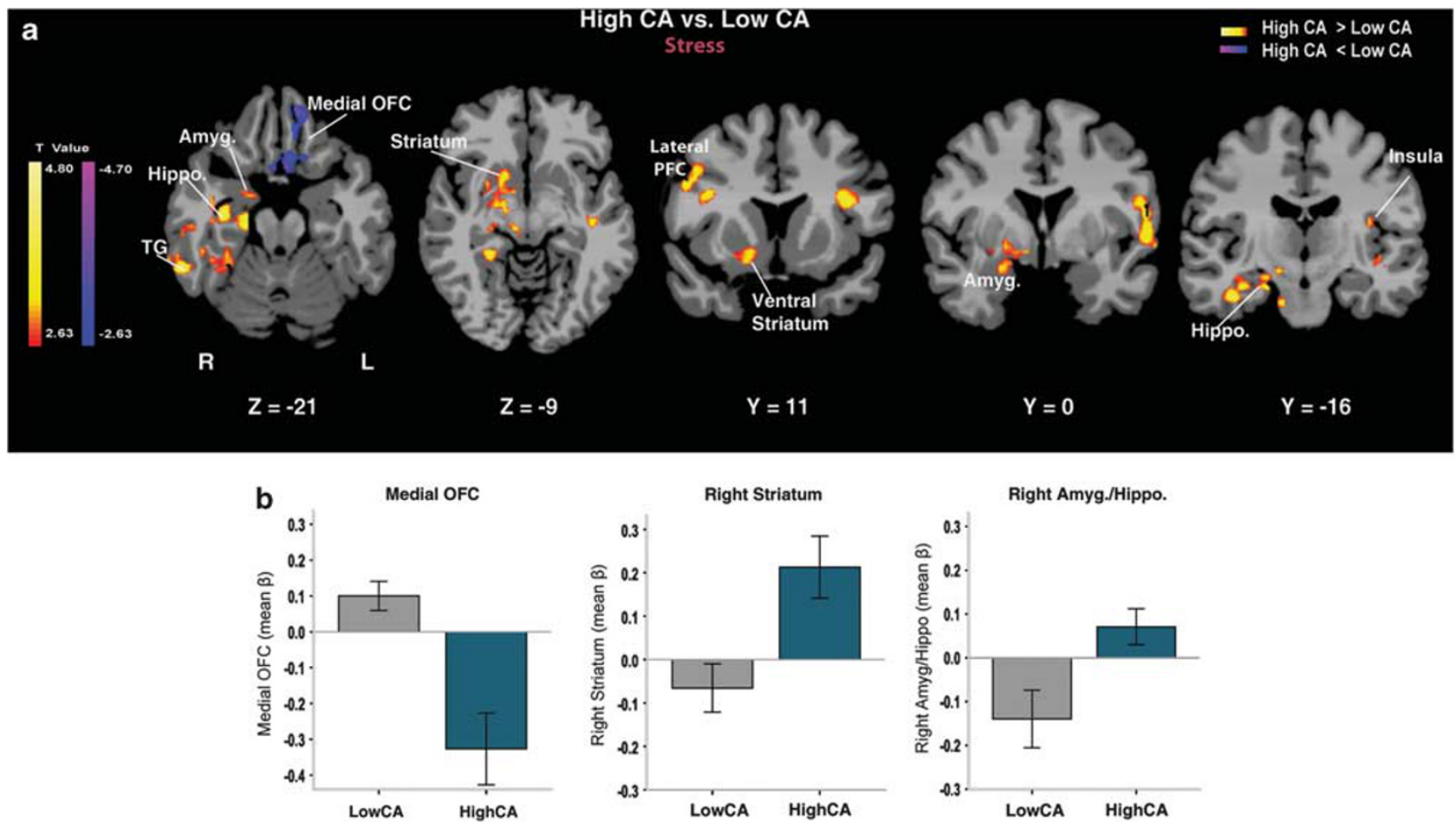

Figure 3 Group differences in stress-induced brain activity in high cumulative adversity (CA) vs low CA group. (a) During stress exposure, brain activity in the high CA group was elevated in the lateral prefrontal cortex (PFC), striatum, amygdala, hippocampus, insula, and superior and inferior temporal lobe, but decreased in the medial orbitofrontal cortex (OFC) relative to the low CA group (whole-brain FWE-corrected, $p<0.0$ I). (b) The bar graphs illustrate group differences in blood oxygenation level-dependent (BOLD) responses in the medial OFC, striatum, and amygdala/hippocampus. L, left; R, right; Amyg., amygdala; Hippo., hippocampus; TG, temporal gyrus. Montreal Neurological Institute (MNI) coordinates were used. 

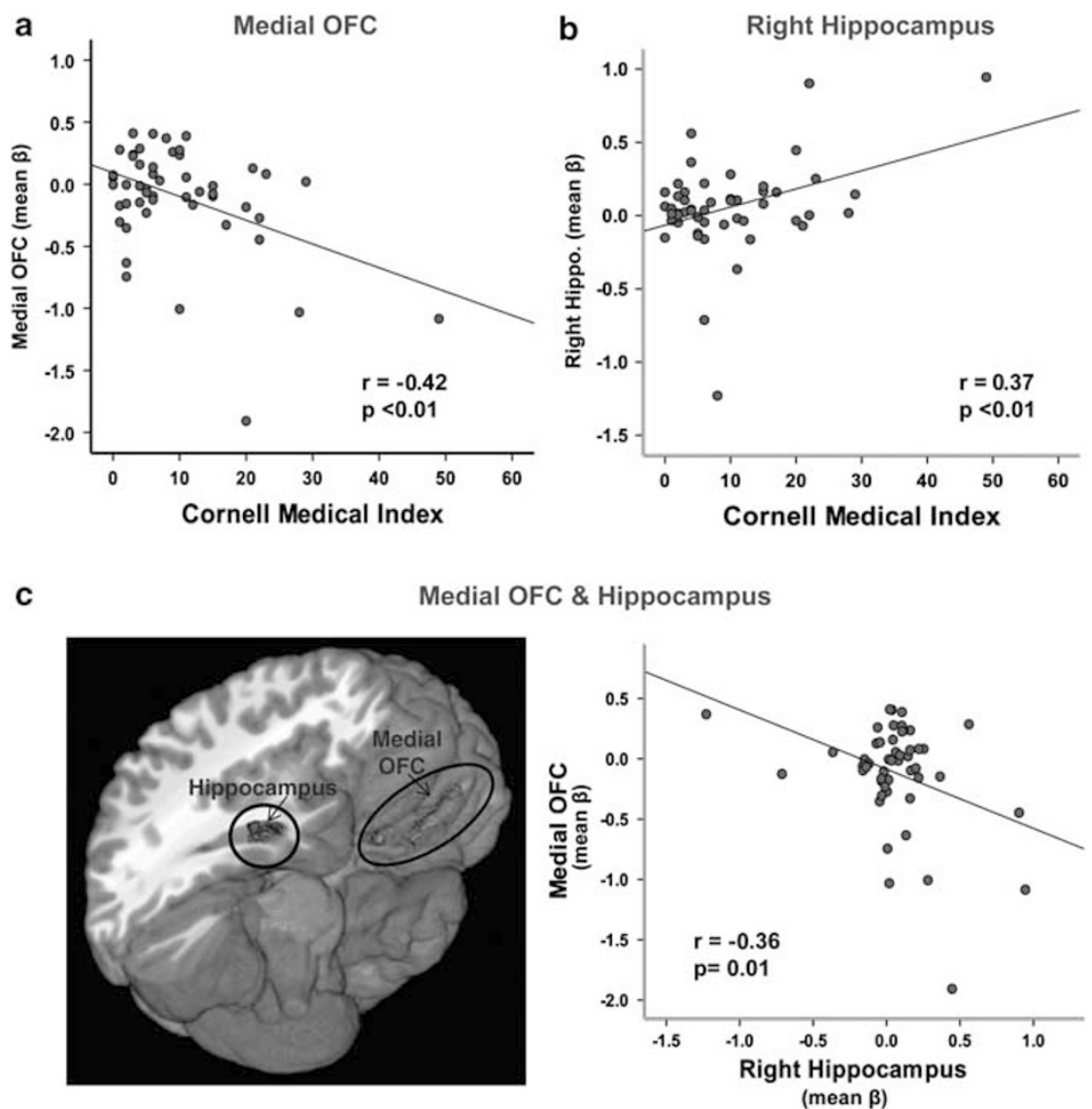

Figure 4 Neural substrates underlying the association between cumulative adversity (CA) and Cornell Medical Index (CMI) health problems in high and low CA individuals. During stress exposure, CMI health problems were (a) negatively correlated with medial orbitofrontal cortex (OFC) activity, but (b) positively correlated with right hippocampal activity. (c) Medial OFC activity was also inversely correlated with right hippocampal activity, suggesting that decreased regulatory function in the medial OFC and disinhibited right hippocampal activity contributes to the link between CA and greater CMI health symptoms. Decreased medial OFC activity and increased right hippocampal activity in high CA individuals relative to low CA individuals identified with whole-brain correction at $p<0.01$ are shown as a color overlay on the three-dimensional surface map.

projections to the frontal cortex (Dias-Ferreira et al, 2009). These results indicate that stress-induced hyperactivity in limbic-striatal regions may reflect sensitized responses to emotional distress in healthy individuals with high CA. During neutral-relaxing imagery, an active control condition reflecting general imagery processing, increased activity in the left parietal lobe (IPL, precuneus) was associated with CA in all subjects, but not in the high/low CA comparison, suggesting a general association pattern with mental imagery in healthy individuals. The left IPL and precuneus are involved in arousal and self-conscious mental process (Cavanna and Trimble, 2006; Singh-Curry and Husain, 2009), suggesting that increased activity in these regions may reflect greater levels of arousal and selfconsciousness during neutral-relaxing imagery in individuals with high CA. It should be noted that altered response in the hypothesized PFC-limbic-striatal circuit was only found during stress exposure, suggesting that the altered PFC-limbic-striatal response is stress-specific and not related to general imagery processing.

In prefrontal regions, increased stress-induced activity in the lateral PFC was significantly associated with CA. Sensory information from limbic-striatal regions are transferred and represented in the lateral PFC (Miller and Cohen, 2001). The lateral PFC integrates this information and engages in prompt cognitive recognition of stress experiences (Miller and Cohen, 2001). It processes cognitive and emotional aspects of the experience and communicates with the medial PFC, a region involved in endogenous physiological and emotional regulation (Diorio et al, 1993; Spencer et al, 2005; Thayer et al, 2012; Urry et al, 2006). Current findings of hyperactive lateral PFC associated with high CA may reflect heightened sensitivity to cognitive processing and detection of stress experiences in these individuals. When high CA individuals were compared with low CA individuals, decreased medial OFC activity was additionally revealed during stress exposure, suggesting a specific neural response to acute stress among those with high levels of CA (see Figure 3; Supplementary Figure S1). The medial OFC has abundant anatomical connections to the amygdala and hippocampus (Carmichael and Price, 1995), allowing top-down regulation of emotional and physiological arousal, including cardiovascular activity and HPA axis responses (Figueiredo et al, 2003; Radley et al, 2006a). Hypofunction in the medial OFC has been associated with emotion regulatory difficulties such as greater anxiety, negative emotion, and impulsivity (Milad and Rauch, 2007; Seo et al, 2008). Taken together, these studies indicate that in high CA individuals, endogenous regulatory control in the medial OFC over emotional and physiological arousal 
during stress may not be effectively implemented, suggesting a greater risk for stress-related health problems.

In our study, greater CMI health problems were associated with hypoactive medial OFC and hyperactive right hippocampal responses to stress, with an inverse relationship between these two regions, indicating a functional interaction between the lower medial OFC and higher right hippocampus during stress in modulating adverse health symptoms. The hippocampus is a key region involved in emotion, memory, and learning (Goosens, 2011). It is highly vulnerable to chronic stress (McEwen, 2002), including sensitivity to sustained glucocorticoid release and damage to the plasticity of the hippocampal nerve cells under prolonged stress exposure (McEwen, 2001). Individuals with long-term trauma and life stress typically show hippocampal volume reduction (Hull, 2002), especially in the right hemisphere (Gianaros et al, 2007). Multiple preclinical studies also demonstrate that repeated chronic stress compromises hippocampal function and hippocampus-associated learning (Nishimura et al, 1999).

The medial PFC, an important region for emotion regulation, has also been found to be impaired following stress exposure. Animal studies showed that repeated restraint stress impairs medial PFC function via dendritic spine loss in rats (Radley et al, 2006b). In humans, the medial PFC was shown to be hypoactive in response to acute stress (Ossewaarde et al, 2011), and a recent study with healthy individuals also showed that high CA and adversity was associated with lower gray matter volume in the medial PFC (Ansell et al, 2012b). The medial OFC has dense anatomical connections with the hippocampus (Carmichael and Price, 1995) and modulates firing of hippocampal neurons (Hyman et al, 2005). It has been suggested that altered medial PFC is involved in the behavioral disruptions associated with hippocampal damage in rats (O'Donnell et al, 2002). Hippocampal damage can also adversely impact medial PFC functions, including neonatal hippocampal lesion associated with decreased dendritic spine density of the PFC pyramidal neurons (Lipska et al, 2001), suggesting a close interaction between the hippocampus and medial PFC.

The medial OFC and hippocampus may influence physical health via their involvement in autonomic, neuroendocrine, and immune functions. For example, the ventromedial PFC (including the medial OFC) modulates stress-related physiological changes via its influence on the hypothalamic paraventricular nucleus, which regulates autonomic nervous system (ANS) and HPA activity (Radley et al, 2006a; Spencer et al, 2005). Specifically, the interaction between the medial PFC and amygdalahippocampal complex has an important role in modulating stress-related ANS activity, including cardiovascular modulation (Thayer et al, 2012). The medial OFC and hippocampus are also likely to modulate immune responses via the HPA axis (Dhabhar, 2003). The medial PFC is a major regulator of the HPA axis (Figueiredo et al, 2003), and the hippocampus has a role in negative feedback of the HPA axis (Sapolsky, 1994) and in the modulation of immune function (Phillips et al, 1999). Additional evidence supports a role for the hippocampus and medial OFC in modulating physical health, such as hippocampal involvement in chronic pain (McEwen, 2001) and interleukin-6 inflammatory function (Marsland et al, 2008), hippocampal damage during cardiac arrest (Sadowski et al, 1999), and decreased OFC blood flow in patients with chronic pain syndrome (Honda et al, 2007). Taken together, current findings indicate a significant role of the medial OFC and hippocampus in regulation of stress and health symptoms, presumably via their effects on the autonomic, neuroendocrine, and immune function involved in homeostasis, supporting the notion that CA-related altered brain responses to stress in these regions may negatively affect physical and psychological health.

It is important to note that CA was retrospectively reported in a structured interview and may have been susceptible to reporting bias and over-reliance on memory. Nonetheless, the CAI has demonstrated validity in large epidemiologic studies that prospectively assessed prediction of psychiatric disorders and physical health conditions (Brown and Turner, 2010; Gayman et al, 2008; Russell et al, 2009; Scott et al, 2008; Turner and Lloyd, 2004). Furthermore, in our data no association was found between CA and STAI anxiety or in-scanner anxiety ratings. Given that anxious tendencies have been closely associated with negative emotional bias (Watson et al, 1988), these results suggest that the objective count of CA is independent of subjective perception or negative emotional bias. Supporting this, we also did not find correlations between CAassociated brain activity in the prefrontal-limbic-striatal regions and scores of a chronic stress subscale specifically designed to capture subjective reactions to adverse events, indicating that current findings are not likely to be driven by subjective response to those events. Furthermore, as significant associations with CA were mainly found in brain response and cardiac autonomic activity measured by increased heart rate, these data suggest that CA significantly influences neural and physiological responses rather than subjective responses in healthy individuals, as suggested in previous work (Ansell et al, 2012b; Thoits, 2010).

In conclusion, the current study identifies CA-related, sensitized prefrontal-limbic-striatal responses to acute stress in non-diseased individuals, and suggests that cumulative experience of adversity affects health problems via altered medial OFC and right hippocampus function during stress. It should be noted that our participants are healthy, community individuals who experienced traumatic or adverse life events, but without current or lifetime PTSD, other psychiatric disorders, or physical illnesses. Although it is possible that the observed stress-induced brain response reflects a protective neural pattern in healthy individuals, the significant associations between activity in the medial OFC/hippocampus and CMI health problems (including both psychological and physiological health symptoms) suggest that altered neural responses in high CA participants may more probably represent the prodromal pattern along a continuum of stress-related pathophysiology that promotes disease risk. To further clarify the exact nature of brain activity association with $\mathrm{CA}$, future research would benefit from including individuals with clinical disorders and also examining whether sensitized responses to stress in vulnerable individuals is predictive of future health problems and development of stress-related diseases. Nonetheless, the current findings have important clinical implications. In the absence of resilience factors and 
in the face of future stressful events, individuals with high CA may be at risk of developing stress-related psychiatric or physical disorders. The findings also suggest the need to further explore the utility of targeting individuals with high $\mathrm{CA}$ and low resilience for developing stress-related primary prevention strategies to reverse the identified neural pathophysiology and stress-related health symptoms, and proactively address their high risk for the development of stress-related disorders.

\section{FUNDING AND DISCLOSURE}

This research was supported by grants from the NIH Roadmap for Medical Research Common Fund and the National Institutes of Drug Abuse (NIDA) and the National Institute of Alcohol Abuse and Alcoholism (NIAAA): UL1DE019586 (RS), R01-AA13892 (RS), PL1-DA024859 (RS), RL1-AA017539 (MP), and K08-DA029641 (EA). Dr Sinha is on the Scientific Advisory Board for Embera Neurotherapeutics. Dr Potenza has consulted for and advised Boehringer Ingelheim, Lundbeck and Ironwood; has received research support from the National Institutes of Health, Veteran's Administration, Mohegan Sun Casino, the National Center for Responsible Gaming, and Forest Laboratories and Psyadon Pharmaceuticals; has participated in surveys, mailings, or telephone consultations related to drug addiction, impulse-control disorders, or other health topics; has consulted for law offices and gambling entities on issues related to addictions or impulse-control disorders; has provided clinical care in the Connecticut Department of Mental Health and Addiction Services Problem Gambling Services Program; has performed grant reviews for the National Institutes of Health and other agencies; has guest-edited journal sections; has given academic lectures in grand rounds, CME events and other clinical or scientific venues; and has generated books or book chapters for publishers of mental health texts.

\section{ACKNOWLEDGEMENTS}

We thank Drs Bruce S McEwen and Keri Tuit for their valuable comments and help with this study.

\section{REFERENCES}

Abrahams N, Alf E (1978). Relative costs and statistical power in the extreme groups approach. Psychometrika 43: 11-17.

Abramson JH (1966). The cornell medical index as an epidemiological tool. Am J Public Health Nations Health 56: 287-298.

Angold A, Erkanli A, Silberg J, Eaves L, Costello EJ (2002). Depression scale scores in 8-17-year-olds: effects of age and gender. J Child Psychol Psychiatry 43: 1052-1063.

Ansell EB, Gu P, Tuit K, Sinha R (2012a). Effects of cumulative stress and impulsivity on smoking status. Hum Psychopharmacol 27: 200-208.

Ansell EB, Rando K, Tuit K, Guarnaccia J, Sinha R (2012b). Cumulative adversity and smaller gray matter volume in medial prefrontal, anterior cingulate, and insula regions. Biol Psychiatry 72: $57-64$.
Borsook D, Becerra L, Carlezon WA Jr, Shaw M, Renshaw P, Elman I et al (2007). Reward-aversion circuitry in analgesia and pain: implications for psychiatric disorders. Eur J Pain 11: 7-20.

Britton JC, Phan KL, Taylor SF, Fig LM, Liberzon I (2005). Corticolimbic blood flow in posttraumatic stress disorder during script-driven imagery. Biol Psychiatry 57: 832-840.

Brown RL, Turner RJ (2010). Physical disability and depression: clarifying racial/ethnic contrasts. J Aging Health 22: 977-1000.

Carmichael ST, Price JL (1995). Limbic connections of the orbital and medial prefrontal cortex in macaque monkeys. J Comp Neurol 363: 615-641.

Cavanna AE, Trimble MR (2006). The precuneus: a review of its functional anatomy and behavioural correlates. Brain J Neurol 129(Pt 3): 564-583.

Costa PT Jr, McCrae RR (1985). Hypochondriasis, neuroticism, and aging. When are somatic complaints unfounded? Am Psychol 40: 19-28.

Craig AD (2009). How do you feel-now? The anterior insula and human awareness. Nat Rev Neurosci 10: 59-70.

Dhabhar FS (2003). Stress, leukocyte trafficking, and the augmentation of skin immune function. Ann NY Acad Sci 992: 205-217.

Dias-Ferreira E, Sousa JC, Melo I, Morgado P, Mesquita AR, Cerqueira JJ et al (2009). Chronic stress causes frontostriatal reorganization and affects decision-making. Science 325: 621-625.

Diorio D, Viau V, Meaney MJ (1993). The role of the medial prefrontal cortex (cingulate gyrus) in the regulation of hypothalamic-pituitary-adrenal responses to stress. J Neurosci 13: 3839-3847.

Duncan JS, Papademetris X, Yang J, Jackowski M, Zeng X, Staib LH (2004). Geometric strategies for neuroanatomic analysis from MRI. Neuroimage 23(Suppl 1): S34-S45.

Figueiredo HF, Bruestle A, Bodie B, Dolgas CM, Herman JP (2003). The medial prefrontal cortex differentially regulates stressinduced c-fos expression in the forebrain depending on type of stressor. Eur J Neurosci 18: 2357-2364.

Fowler RL (1992). Using the extreme groups strategy when measures are not normally distributed. Appl Psychol Measure 16: 249-259.

Gayman MD, Turner RJ, Cui M (2008). Physical limitations and depressive symptoms: exploring the nature of the association. J Gerontol B Psychol Sci Soc Sci 63: S219-S228.

Gianaros PJ, Jennings JR, Sheu LK, Greer PJ, Kuller LH, Matthews KA (2007). Prospective reports of chronic life stress predict decreased grey matter volume in the hippocampus. Neuroimage 35: 795-803.

Goosens KA (2011). Hippocampal regulation of aversive memories. Curr Opin Neurobiol 21: 460-466.

Henshall JM, Goddard ME (1999). Multiple-trait mapping of quantitative trait loci after selective genotyping using logistic regression. Genetics 151: 885-894.

Holmes CJ, Hoge R, Collins L, Woods R, Toga AW, Evans AC (1998). Enhancement of MR images using registration for signal averaging. J Comput Assist Tomogr 22: 324-333.

Honda T, Maruta T, Takahashi K (2007). Brain perfusion abnormality in patients with chronic pain. Keio J Med 56: 48-52.

Hull AM (2002). Neuroimaging findings in post-traumatic stress disorder. Systematic review. Br J Psychiatry 181: 102-110.

Hyman JM, Zilli EA, Paley AM, Hasselmo ME (2005). Medial prefrontal cortex cells show dynamic modulation with the hippocampal theta rhythm dependent on behavior. Hippocampus 15: 739-749.

Jastreboff AM, Sinha R, Lacadie C, Small DM, Sherwin RS, Potenza MN (2013). Neural correlates of stress- and food cue-induced food craving in obesity: association with insulin levels. Diabetes Care 36: 394-402. 
Karst H, Joels M (2003). Effect of chronic stress on synaptic currents in rat hippocampal dentate gyrus neurons. $J$ Neurophysiol 89: 625-633.

Lanteaume L, Khalfa S, Regis J, Marquis P, Chauvel P, Bartolomei F (2007). Emotion induction after direct intracerebral stimulations of human amygdala. Cereb Cortex 17: 1307-1313.

Li CS, Sinha R (2008). Inhibitory control and emotional stress regulation: neuroimaging evidence for frontal-limbic dysfunction in psycho-stimulant addiction. Neurosci Biobehav Rev 32: 581-597.

Lipska BK, Khaing ZZ, Weickert CS, Weinberger DR (2001). BDNF mRNA expression in rat hippocampus and prefrontal cortex: effects of neonatal ventral hippocampal damage and antipsychotic drugs. Eur J Neurosci 14: 135-144.

Liston C, McEwen BS, Casey BJ (2009). Psychosocial stress reversibly disrupts prefrontal processing and attentional control. Proc Natl Acad Sci USA 106: 912-917.

Liston C, Miller MM, Goldwater DS, Radley JJ, Rocher AB, Hof PR et al (2006). Stress-induced alterations in prefrontal cortical dendritic morphology predict selective impairments in perceptual attentional set-shifting. J Neurosci 26: 7870-7874.

Lloyd DA, Turner RJ (2008). Cumulative lifetime adversities and alcohol dependence in adolescence and young adulthood. Drug Alcohol Depend 93: 217-226.

Marsland AL, Gianaros PJ, Abramowitch SM, Manuck SB, Hariri AR (2008). Interleukin-6 covaries inversely with hippocampal grey matter volume in middle-aged adults. Biol Psychiatry 64: 484-490.

Martijena ID, Rodriguez Manzanares PA, Lacerra C, Molina VA (2002). Gabaergic modulation of the stress response in frontal cortex and amygdala. Synapse 45: 86-94.

McEwen BS (1998). Stress, adaptation, and disease. Allostasis and allostatic load. Ann NY Acad Sci 840: 33-44.

McEwen BS (2001). Plasticity of the hippocampus: adaptation to chronic stress and allostatic load. Ann NY Acad Sci 933: 265-277.

McEwen BS (2002). Sex, stress and the hippocampus: allostasis, allostatic load and the aging process. Neurobiol Aging 23: 921-939.

McEwen BS, Stellar E (1993). Stress and the individual. Mechanisms leading to disease. Arch Intern Med 153: 2093-2101.

Milad MR, Rauch SL (2007). The role of the orbitofrontal cortex in anxiety disorders. Ann NY Acad Sci 1121: 546-561.

Miller EK, Cohen JD (2001). An integrative theory of prefrontal cortex function. Annu Rev Neurosci 24: 167-202.

Morris JS, Ohman A, Dolan RJ (1999). A subcortical pathway to the right amygdala mediating 'unseen' fear. Proc Natl Acad Sci USA 96: $1680-1685$.

Nishimura J, Endo Y, Kimura F (1999). A long-term stress exposure impairs maze learning performance in rats. Neurosci Lett 273: 125-128.

Nolfe E (2003). XMedCon-An open-source medical image conversion toolkit. Eur J Nuclear Med 30(Supp2): S246; TP39.

O'Donnell P, Lewis BL, Weinberger DR, Lipska BK (2002). Neonatal hippocampal damage alters electrophysiological properties of prefrontal cortical neurons in adult rats. Cereb Cortex 12: $975-982$

Orr SP, Pitman RK, Lasko NB, Herz LR (1993). Psychophysiological assessment of posttraumatic stress disorder imagery in World War II and Korean combat veterans. J Abnorm Psychol 102: $152-159$.

Ossewaarde L, Qin S, Van Marle HJ, van Wingen GA, Fernandez G, Hermans EJ (2011). Stress-induced reduction in reward-related prefrontal cortex function. Neuroimage 55: 345-352.

Panksepp J, Nelson E, Bekkedal M (1997). Brain systems for the mediation of social separation-distress and social-reward. Evolutionary antecedents and neuropeptide intermediaries. Ann NY Acad Sci 807: 78-100.
Perlmutter M, Nyquist L (1990). Relationships between selfreported physical and mental health and intelligence performance across adulthood. J Gerontol 45: P145-P155.

Phelps EA (2004). Human emotion and memory: interactions of the amygdala and hippocampal complex. Curr Opin Neurobiol 14: 198-202.

Phillips LM, Simon PJ, Lampson LA (1999). Site-specific immune regulation in the brain: differential modulation of major histocompatibility complex (MHC) proteins in brainstem vs hippocampus. J Comp Neurol 405: 322-333.

Potenza MN, Hong KI, Lacadie CM, Fulbright RK, Tuit KL, Sinha R (2012). Neural correlates of stress-induced and cue-induced drug craving: influences of sex and cocaine dependence. Am J Psychiatry 169: 406-414.

Radley J, Arias C, Sawchenkole PE (2006a). Regional differentiation of the medial prefrontal cortex in regulating adaptive responses to acute emotional stress. J Neurosci 26: 12967-12976.

Radley JJ, Rocher AB, Miller M, Janssen WG, Liston C, Hof PR et al (2006b). Repeated stress induces dendritic spine loss in the rat medial prefrontal cortex. Cereb Cortex 16: 313-320.

Romens SE, Abramson LY, Alloy LB (2009). High and low cognitive risk for depression: stability from late adolescence to early adulthood. Cognit Ther Res 33: 480-498.

Rossi S, De Chiara V, Musella A, Kusayanagi H, Mataluni G, Bernardi G et al (2008). Chronic psychoemotional stress impairs cannabinoid-receptor-mediated control of GABA transmission in the striatum. J Neurosci 28: 7284-7292.

Russell D, Turner RJ, Joiner TE (2009). Physical disability and suicidal ideation: a community-based study of risk/protective factors for suicidal thoughts. Suicide Life Threat Behav 39: 440-451.

Sadowski M, Wisniewski HM, Jakubowska-Sadowska K, Tarnawski M, Lazarewicz JW, Mossakowski MJ (1999). Pattern of neuronal loss in the rat hippocampus following experimental cardiac arrest-induced ischemia. J Neurol Sci 168: 13-20.

Sapolsky RM (1994). The physiological relevance of glucocorticoid endangerment of the hippocampus. Ann NY Acad Sci 746: 294-304 Discussion 304-297.

Schultz W (2006). Behavioral theories and the neurophysiology of reward. Annu Rev Psychol 57: 87-115.

Scott KM, Von Korff M, Alonso J, Angermeyer MC, Benjet C, Bruffaerts $\mathrm{R}$ et al (2008). Childhood adversity, early-onset depressive/anxiety disorders, and adult-onset asthma. Psychosom Med 70: 1035-1043.

Seeman TE, Crimmins E, Huang $\mathrm{MH}$, Singer B, Bucur A, Gruenewald $\mathrm{T}$ et al (2004). Cumulative biological risk and socio-economic differences in mortality: MacArthur studies of successful aging. Soc Sci Med 58: 1985-1997.

Seo D, Jia Z, Lacadie CM, Tsou KA, Bergquist K, Sinha R (2011). Sex differences in neural responses to stress and alcohol context cues. Hum Brain Mapp 32: 1998-2013.

Seo D, Lacadie CM, Tuit K, Hong KI, Constable RT, Sinha R (2013). Disrupted ventromedial prefrontal function, alcohol craving, and subsequent relapse risk. JAMA Psychiatry 70: 727-739.

Seo D, Patrick CJ, Kennealy PJ (2008). Role of serotonin and dopamine system interactions in the neurobiology of impulsive aggression and its comorbidity with other clinical disorders. Aggress Violent Behav 13: 383-395.

Sheehan P (1967). A shortened version of the Bett's questionnaire upon mental imagery. J Clin Psychol 23: 386-389.

Singh-Curry V, Husain M (2009). The functional role of the inferior parietal lobe in the dorsal and ventral stream dichotomy. Neuropsychologia 47: 1434-1448.

Sinha R (2008). Chronic stress, drug use, and vulnerability to addiction. Ann NY Acad Sci 1141: 105-130.

Sinha R (2009). Modeling stress and drug craving in the laboratory: implications for addiction treatment development. Addict Biol 14: 84-98. 
Sinha R, Lacadie C, Skudlarski P, Wexler BE (2004). Neural circuits underlying emotional distress in humans. Ann NY Acad Sci 1032: 254-257.

Spencer SJ, Buller KM, Day TA (2005). Medial prefrontal cortex control of the paraventricular hypothalamic nucleus response to psychological stress: possible role of the bed nucleus of the stria terminalis. J Comp Neurol 481: 363-376.

Spielberger CD, Gorsuch RL, Lushene R, Vagg PR, Jacobs GA (1983). Manual for the State-Trait Anxiety Inventory. Consulting Psychologists Press: Palo Alto, CA.

Stroud LR, Salovey P, Epel ES (2002). Sex differences in stress responses: social rejection versus achievement stress. Biol Psychiatry 52: 318-327.

Thayer JF, Ahs F, Fredrikson M, Sollers JJ 3rd, Wager TD (2012). A meta-analysis of heart rate variability and neuroimaging studies: implications for heart rate variability as a marker of stress and health. Neurosci Biobehav Rev 36: 747-756.

Thoits PA (2010). Stress and health: major findings and policy implications. J Health Soc Behav 51(Suppl): S41-S53.

Turner R, Wheaton B, Lloyd D (1995). The epidemiology of social stress. Am Socio Rev 60: 104-125.
Turner RJ, Gil AG (2002). Psychiatric and substance use disorders in South Florida: racial/ethnic and gender contrasts in a young adult cohort. Arch Gen Psychiatry 59: 43-50.

Turner RJ, Lloyd DA (2004). Stress burden and the lifetime incidence of psychiatric disorder in young adults: racial and ethnic contrasts. Arch Gen Psychiatry 61: 481-488.

Urry HL, van Reekum CM, Johnstone T, Kalin NH, Thurow ME, Schaefer HS et al (2006). Amygdala and ventromedial prefrontal cortex are inversely coupled during regulation of negative affect and predict the diurnal pattern of cortisol secretion among older adults. J Neurosci 26: 4415-4425.

Vink M, Kahn RS, Raemaekers $M$, van den Heuvel M, Boersma M, Ramsey NF (2005). Function of striatum beyond inhibition and execution of motor responses. Hum Brain Mapp 25: 336-344.

Watson D, Clark LA, Carey G (1988). Positive and negative affectivity and their relation to anxiety and depressive disorders. J Abnorm Psychol 97: 346-353.

Xiong J, Gao J-H, Lancaster J, Peter TF (1995). Clustered pixels analysis for functional MRI activation studies of the human brain. Hum Brain Mapp 3: 287-301.

Supplementary Information accompanies the paper on the Neuropsychopharmacology website (http://www.nature.com/npp) 